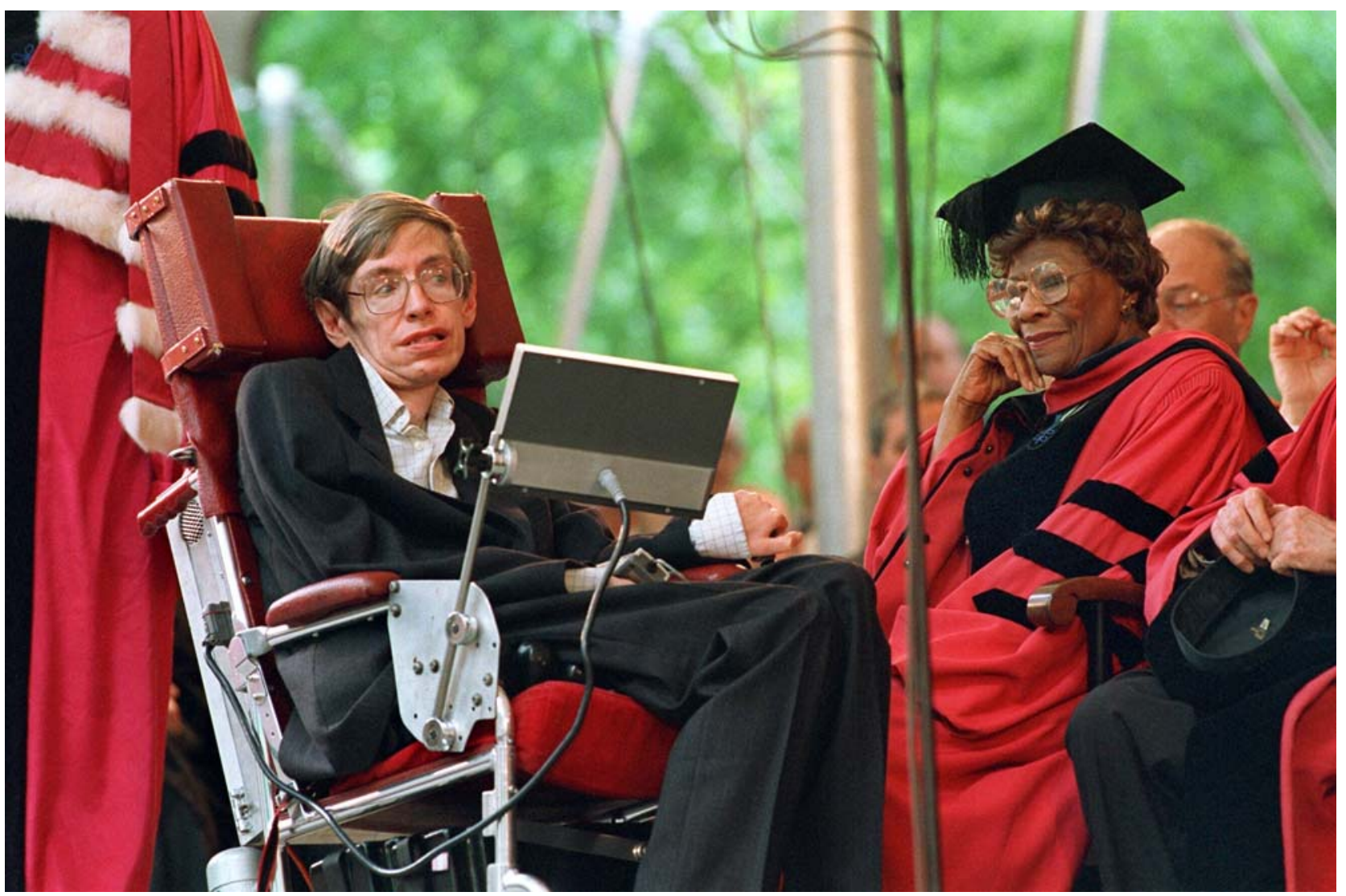

Stephen Hawking mit Ella Fitzgerald bei der Entgegennahme der Harvard-Ehrendoktorwürde (Foto: dpa)

\title{
Ihr Auftritt, Mrs. Theorem!
}

\section{von Ivo Schneider}

Gerhard Schröders Absicht, mit Hilfe von nach amerikanischem Vorbild ausgestellten Greencards Computerspezialisten aus dem Ausland, vor allem aus Indien, zu holen, traf auf die unterschiedlichsten Reaktionen. Sie reichen von rassistisch begründeter Ablehnung bis zu uneingeschränktem Beifall von denen, die wissen, dass der längst deutliche Rückstand gegenüber anderen Ländern nicht kurzfristig mit deutschen Kräften überbrückbar ist, sondern sich ohne eine solche Maßnahme rasch vergrößern würde.

Die angesprochene Situation wird von Fachleuten mit einer spezifisch bundesrepublikanischen intellektuellen Befindlichkeit in Zusammenhang gebracht. Danach ist Deutschland einem seit Jahren kolportierten Bonmot zufolge das einzige Land, in dem man durch das Bekenntnis mangelhafter Begabung oder mangelhafter Kenntnisse in Mathematik gesellschaftliches Prestige gewinnen kann. Die mangelnde Akzeptanz der Mathematik und der sich ihrer Sprache und Methoden bedienenden Wissenschaften in der deutschen Öffentlichkeit wurzelt in Werthaltungen des deutschen Bildungsbürgertums, die sich bis ins 19. Jahrhundert zurückverfolgen lassen. Weitgehend unterdrückt während einer Phase wachsender Fortschrittsgläubigkeit in den Jahrzehnten vor dem Er- sten Weltkrieg wurden diese Werte nach der Niederlage von 1918 wieder offen propagiert. Oswald Spenglers Rede von der kalten Hand der Rationalität steht dafür ebenso wie die von der Kultusbürokratie während der Weimarer Republik durchgesetzten drastischen Kürzungen des Stundendeputats in Mathematik und Naturwissenschaften an deutschen Gymnasien. Das Virus einer solchen gegen die Mathematik gerichteten Haltung konnte sich nach der Machtübernahme durch das NS-Regime offiziell mit einem schon lange vorher vorhandenen Antisemitismus verbinden, um z. B. Einsteins Relativitätstheorie unter Hinweis auf ihre abstrakten mathematischen Strukturen als unanschaulich, dem gesunden Volksempfinden und Menschenverstand widersprechend, steril und damit 
jüdisch zu diffamieren. In der nun schon über ein halbes Jahrhundert alten Bundesrepublik ist trotz aller Veränderungen und trotz aller Meinungs- und Haltungsvielfalt das bildungsbürgerliche Erbe noch immer erkennbar in der hartnäckigen Weigerung, den Bereich Mathematik, Naturwissenschaften und Technik als legitimen Bestandteil der Kultur anzuerkennen.

Wer die während des kalten Krieges entstandene zunehmend kritische Haltung der amerikanischen Gesellschaft gegenüber Naturwissenschaft und Technik oder Percy S. Snows nun auch schon in die Jahre gekommene Unterscheidung zwischen den beiden Kulturen der Natur- und Geisteswissenschaften als Beleg für vergleichbare Verhältnisse in der englischsprachigen Welt versteht, wird sich jetzt zusammen mit den New Yorker Theaterkritikern verwundert die Augen reiben: Sie werden zu Trauzeugen einer überraschenden Eheschließung. In der amerikanischen Theaterund Filmwelt, vor allem sichtbar in New York, häufen sich Stücke, die mit der Welt der Physik und der Mathematik zu tun haben. Michael Frayns Copenhagen, das um den Besuch Heisenbergs bei Niels Bohr im Jahr 1942 zentriert ist und dabei auch die damals aktuellen Probleme der Quantenmechanik ins Spiel bringt, ist nach seinem von der Londoner Kritik gefeierten Start das inzwischen bekannteste der mit Physik befassten Stücke in New York; dazu kommen Space von Tina Landau im Public Theater, in dem es u. a. um Kommunikation mit Wesen von weit entfernten Galaxien des Kosmos geht, Moving Bodies, eine Biographie des Physikers Richard Feynman von Arthur Giron im Ensemble Studio Theater, sowie Now Then Again von der erst 30-jährigen Penny Penniston, das nach seiner Premiere im Bailiwick Repertory bis Ende Mai am Ivanhoe Theater in Chicago lief und von der Romanze zwischen einem jungen Physiker und einer Physikstudentin berichtet, deren zeitliche Entwicklung bis zu einem bestimmten Zeitpunkt im dritten Akt und dann wieder zurück Kenner an ein Feynman-Diagramm erinnerte.

Konnte man das nach Beendigung des kalten Krieges wieder erwachte Interesse der Theaterwelt an der Physik mit den an das Manhattan Projekt anschließenden Entwicklungen, aber auch mit den durch das Internet und das Handy gegebenen technisch vermittelten neuen Komminikationsmöglichkeiten verbinden, so versagt ein solcher Erklärungsversuch bei den Theaterstücken, die sich der Mathematik und vor allem ihrer reinsten Ausprägung, der Zahlentheorie, widmen. Hinter Hypatia von Mac Wellmann, einer im Soho Rep and Ridge Theatre inszenierten Geschichte von Leben und Werk der von Christen im spätantiken Alexandria erschlagenen Mathematikerin Hypatia, die im Verlauf der Aufführung alle paar Jahrhun- derte auf wunderbare Weise wieder erscheint, um die Welt mit einer neuen Gabe ihrer Einbildungskraft zu bedenken, steht natürlich auch das von der Frauenbewegung ausgehende Interesse an von Frauen erbrachten Leistungen auf Gebieten, die wie die Mathematik nicht gerade als weibliche Domänen gelten. In dieselbe Richtung geht der 1997 unter der Regie von Lynn Hershman Leeson in den USA gedrehte Film Conceiving Ada, der sich an der Tochter Lord Byrons, der ersten Anwenderin eines Rechnerprogramms, Lady Ada Lovelace, orientiert. Im Film entwickelt Emmy, ein Computerfreak unserer Zeit, eine Maschine, die über zwei Jahrhunderte hinweg den direkten Kontakt zu Ada Lovelace ermöglicht, um damit deren mathematische Ideen, vor sie durch Adas vorzeitigen Tod für immer verloren gehen, dem Vergessen zu entreißen.

„Sie sagte, dass weder sie noch irgendeiner ihrer Bekannten die ganze Wissenschaft in dem Stück mochten, und dass es sich auf dem Broadway nicht lange halten würde, wenn ich das nicht rausnähme."

Michael Frayn

In The five hysterical girls theorem von Rinne Groff am Target Margin Theater, geht es um Zahlentheoretisches, unter anderem um sehr große Zahlen wie die 21-stellige Zahl $2^{67}-1$, die sich als nichtprim erweist, aber deren weitere Eigenschaften ebenso im Dunkel bleiben wie deren Funktion, den Selbstmord eines jungen Mathematikers auszulösen. Aufregender ist Proof von David Auburn im Manhattan Theater Club, in dem es am Beispiel von zahlentheoretischen Sätzen und deren Beweise um ein Verständnis der Arbeitsweise von Mathematikern geht. Obwohl sich Auburn für die Bühnenfassung in New York von Mathematikern des Courant Instituts beraten ließ, hatte die Kritik mehr Einblick in die Arbeit von Mathematikern erwartet als es die wenigen, den Protagonisten offenbar mühelos möglichen Geniestreiche tun in einer Geschichte, die sich um die hochbegabte Tochter eines Mathematikprofessors dreht. Festzuhalten ist jedenfalls das Bemühen des Autors um eine möglichst authentische Darstellung der zur Welt der Mathematik gehörigen menschlichen Seite. Darum geht es auch in dem unter der Regie von Gus van Sant entstandenen Film Good Will Hunting, der zumindest die der Mathematik üblicherweise abgesprochene emotionale Dimension zu erfassen sucht. Er berichtet über die Beziehungen eines mit der FieldsMedal ausgezeichneten Mathematik-Professors an einer amerikanischen Eliteuniversität zu einem ihm wegen einer Straftat anvertrauten mathematisch hochbegabten jungen Mann. 
Außerdem ist in Kürze die Inszenierung eines $\mathrm{Mu}$ sicals ebenfalls mit dem Titel Proof von Joshua Rosenblum und Sydney Lessner über den Mathematiker Andrew Wiles durch die New York Theater Company zu erwarten, das den erst 1995 erbrachten Beweis des sogenannten großen Fermatschen Satzes eben durch Andrew Wiles feiert. Der Beweis, der, nachdem in einer früheren Fassung eine Lücke festgestellt worden war, in seiner zweiten Fassung immerhin 130 Druckseiten beanspruchte, ist so aufwendig, dass ihn bis heute nur verhältnismäßig wenige Fachmathematiker bis in die kleinsten Details durchgearbeitet haben. Dennoch hat die Veröffentlichung des Beweises die Redaktionen von Zeitungen in aller Welt beschäftigt und für kurze Zeit größtes öffentliches Interesse beansprucht. Hintergrund dafür ist offenbar der Umstand, dass sich die besten Mathematiker seit rund 350 Jahren vergeblich darum bemüht hatten, den Beweis für diesen auf einem Buchrand notierten Satz von Fermat zu finden.

"It's fun to write about smart people."

David Auburn

Die Liste der Beispiele für die neue Verbindung zwischen Mathematik und ihren wichtigsten Anwendungsgebieten einerseits und der Theater- und Filmwelt andererseits in den USA könnte fortgesetzt und auf den Bereich der Literatur ausgedehnt werden. Ähnliche Beispiele finden sich in europäischen Ländern vor allem in England. Dort wurde bereits Ende 1986 Breaking the Code, die Rolle von Alan Turing bei der Entschlüsselung des Codes der deutschen
Enigma, im Londoner Haymarket Theatre uraufgeführt, um dann in den folgenden Jahren in einer Reihe anderer Länder gezeigt zu werden. In England wird auch demnächst ein Stück über den dort als Newton unserer Zeit apostrophierten Stephen Hawking und dessen Vorstellungen über Zeit von Robin Hawdon inszeniert werden.

Wie anders das Interesse an solchen Stücken in der Bundesrepublik aussieht, zeigt das Beispiel des in England und den USA so erfolgreichen Copenhagen von Michael Frayn, in dessen Mittelpunkt ja der deutsche Physiker Werner Heisenberg steht. Das Stück wurde, von der deutschen Öffentlichkeit nahezu unbemerkt, 1999 an einigen abgelegenen kleinen deutschen Bühnen von einem Tourneetheaterunternehmen unter der Regie von Fred Berndt, der an Peter Steins Berliner Schaubühne groß wurde, aufgeführt. Einige Dramaturgen deutscher Theater hatten daraufhin den Text der deutschen Fassung von dem zuständigen Theaterverlag angefordert, aber offenbar nach der Lektüre jedes Interesse an einer Aufführung verloren. Auch die einzige Ausnahme Düsseldorf, wo das Stück in nächster Zeit neu inszeniert werden soll, ändert nichts an der deutschen Befindlichkeit, wonach sich die Welt der Mathematik und der menschlicher Emotionen in deutschen Theatern nicht begegnen.

\author{
Anschrift des Autors \\ Prof. Dr. Ivo Schneider \\ Fakultät für Sozialwissenschaften \\ Universität der Bundeswehr München \\ 85577 Neubiberg \\ Ivo.Schneider@unibw-muenchen.de
}

It's not breaking the code that matters - it's where you go from there.

Alan Turing 